\title{
Aerobic capacity of adults with intellectual disabilities
}

\author{
Krystyna Gawlik' ${ }^{1}$ Anna Zwierzchowska', Beata Manowska², Diana Celebańska' \\ ${ }^{1}$ Department of Correction and Special Education, Academy of Physical Education, Katowice, Poland \\ 2 Department of Physiology, Academy of Physical Education, Katowice, Poland
}

Krystyna Gawlik, Anna Zwierzchowska, Beata Manowska, Diana Celebańska. Aerobic capacity of adults with intellectual disabilities. Ann Agric Environ Med. 2017; 24(1): 117-120. doi: 10.5604/12321966.1233999

\begin{abstract}
Introduction. Physical fitness is a resultant of the efficiency of adaptive mechanisms for physical effort. People with intellectual disabilities may exhibit limited adaptive capacities, not only regarding their mental development, but also physiological, social and emotional development. Dysfunctions of the central nervous system observed in individuals with intellectual disabilities cause difficulties in gaining movement experience as well as problems with coordination and kinesthetic sense. Thus, intellectual disability might cause low physical activity and, consequently, low physical fitness.

Objective. The aim of the present investigations was to evaluate aerobic capacity of intellectually disabled adults and determine its potential relationships with the degree of intellectual disability, somatic parameters, age and gender.

Materials and method. The study group consisted of 85 intellectually disabled adults aged 20 to 40 years. The investigations were based on direct observation of the participants. The following somatic parameters were measured: body height and weight, waist and hip circumference, body fat percentage, body mass index and waist-to-hip ratio. Physical fitness was evaluated based on PWC170; VO2max was also calculated. Qualitative assessment of physical fitness level was carried out using Astrand's classification (with respect to age and gender).

Results. $30 \%$ of women and $46.3 \%$ of men had very low level of aerobic capacity. Absolute values of $\mathrm{PWC}_{170}$ and $\mathrm{VO}_{2 \max }$ were significantly higher in men. However, relative values expressed in millilitres of oxygen per kilogram of body weight did not reveal statistically significant inter-gender differences. Age and degree of intellectual disability did not significantly influence the level of physical fitness. Multiple regression analysis to estimate $\mathrm{VO}_{2 \mathrm{max}} / \mathrm{kg}$ revealed a negative correlation with \%FAT and WHR.

Conclusions. 1. A large sample of the study population ( $30 \%$ female, $46.3 \%$ male) showed very low levels of aerobic capacity. 2. Our investigations did not demonstrate a relationship between physical fitness and age or the degree of intellectual disability. Gender turned out to be a differentiating factor but only for the absolute PWC $\mathrm{C}_{170}$ and VO2max. 3. The level of physical fitness was significantly related to somatic parameters including body mass, waist and hips circumference, percentage of body fat, BMI and WHR.
\end{abstract}

\section{- Key words}

aerobic capacity, intellectual disabilities, adults people

\section{INTRODUCTION}

Physical fitness is a resultant of the efficiency of adaptive mechanisms for physical effort. The fitness level depends on physiological, biomechanical and psychological factors. Higher levels of physical fitness enable an individual to perform work with less significant engagement of the whole organism and minimize exercise-induced disturbances to homeostasis. People with intellectual disabilities (ID) may exhibit limited adaptive capacities, not only regarding their mental development, but also physiological, social and emotional developments which are highly related. Dysfunctions of the central nervous system observed in individuals with intellectual disabilities cause difficulties in gaining movement experience as well as problems with coordination and kinesthetic sense. Thus, intellectual disability might cause low physical activity and, consequently, low physical fitness. At the same time, however, irrespective of the wide range of the clinical symptoms of intellectual disability, physical activity constitutes an invaluable

Address for correspondence: Krystyna Gawlik, Department of Correction and Special Education, Academy of Physical Education, Katowice, Poland E-mail: k.gawlik@awf.katowice.pl

Received: 11 June 2013; accepted: 27 March 2014; first published on December, 2016 and indispensable means to alleviate the results thereof. Appropriate intensity and levels of physical activity not only help improve the physiological function but also support the psycho-social rehabilitation. Regular physical activity has comprehensive and complex effects on the ability of intellectually disabled individuals to adapt and carry on everyday life activities as well as on their personal, social and professional functioning. Thus, physical activity should constitute the primary rehabilitation strategy in the intellectually disabled. However, the majority of research reports seem to indicate insufficient physical activity or even lack of any physical activity of these individuals $[1,2$, $3,4,5,6]$.

The researchers investigating physical activity of intellectually disabled individuals generally believe that the activity is insufficient and require effective promotion to fulfil the goals of professional prophylaxis.

\section{OBJECTIVE}

The aim of the present investigations was to evaluate aerobic capacity of intellectually disabled individuals. The following research questions were formed: 
What is the aerobic capacity of the intellectually disabled individuals relative to the general population?

Do the severity of intellectual disability as well as age and gender cause differences in the levels of aerobic capacity?

Is there an association between somatic parameters and aerobic capacity of the intellectually disabled?

\section{MATERIALS AND METHOD}

Ninety-five individuals with intellectual disabilities, participants of an occupational therapy workshop, entered the study. Exercise test was completed by 85 study subjects; the remaining individuals were not able to pedal at the preset work load $(60 \mathrm{rpm})$ or were excluded due to pre-exercise arterial blood pressure elevation or lower limb muscle spasm.

The participants were between 20 and 40 years of life. There were 44 women and 41 men with mild, moderate and considerable intellectual disability (13, 35 and 37, respectively). Group I (GR I) comprised participants aged 20 to 29 years while Group II (GR II) consisted of intellectually disabled individuals aged 30 to 40 years.

The investigations were based on direct observation of the participants. The following somatic parameters were measured: body height and weight, waist and hip circumference (WC), body fat percentage (\%FAT), body mass index (BMI) and waist-to-hip ratio (WHR). Percentage of body fat was measured using Tanita bioelectric impedance analysis (Tanita-BIA).

Exercise capacity was determined via exercise testing which was conducted using a Monark 828e cycle ergometer. Each participant performed two exercise trials at two submaximal workloads. The required pedal cadence was $60 \mathrm{rpm}$. Workload adjustments were based on body mass, age and gender. Heart rate $(\mathrm{HR})$ was continuously monitored with a sport-tester. $\mathrm{PWC}_{170}$ was calculated according to the formula:

$$
P W C_{170}=N_{1}-N_{2} \bullet \frac{170-f_{1}}{f_{1}-f_{2}}
$$

where: N1- workload one, N2- for workload two, $\mathrm{f}_{1}$ - heart rate at minute 5 of workload one, $\mathrm{f}_{2}$ - heart rate at minute 5 of workload two [7]. PWC $_{170}$ estimates the working capacity at a heart rate of 170 beats per minute and is highly correlated with $\mathrm{VO}_{2 \max }$. Maximum oxygen uptake was calculated from the following formula:

$$
\mathrm{VO}_{2 \max }=1.7 \times \mathrm{PWC}_{170} \mathrm{kgm} / \mathrm{min}+1240
$$

Absolute $\mathrm{VO}_{2 \max }$ values were expressed per kilogram body mass. Based on the relative rate in millilitres of oxygen per kilogram of body weight per minute (with respect to age and gender) qualitative assessment of physical fitness level was carried out. Using Astrand's classification, each participant's fitness level was classified as very low (1), low (2), average (3), high (4) or very high (5) [7].

Statistics. The statistics produced for each of quantitative variables were mean, median, maximum, minimum, standard deviation, skewness and kurtosis. The Kolmogorov-Smirnov test was used to determine whether the data had a normal distribution. Crosstabulation tables were used for qualitative variables analysis according to the categories thereof. The relationship between physical fitness and gender, age and the degree of intellectual disability were verified using a two-way analysis of variance and Tukey HSD for post-hoc analysis. The relationship between the level of physical fitness and the degree of intellectual disability, gender and age was determined with a chi-square test for independence. Multiple regression analysis was used to estimate the effects of somatic parameters on physical fitness. One-way analysis of variance was performed in order to determine the relationship between aerobic capacity, and somatic features standardized in groups by gender and age. A somatic profile was established based on standardized values and compared to physical fitness levels.

The presented investigations constitute a part of a research project 'Lifestyle and the risk of civilization diseases in adults with intellectual disabilities' carried out by the Department of the Theory and Methodology of Physical Education of the Jerzy Kukuczka Academy of Physical Education in Katowice. The research had approval of the Bioethics Committee of the Academy of Physical Education. All participants and their legal guardians obtained comprehensive information about the aim and methods of the investigations and gave written consent to participate in the study. Participants had the right to withdraw from the study at any time.

\section{RESULTS}

Somatic and aerobic capacity parameters are presented in Tables 1 and 2.

\begin{tabular}{|c|c|c|c|c|c|c|c|c|}
\hline & \multicolumn{2}{|c|}{ Mean } & \multicolumn{2}{|c|}{ Min } & \multicolumn{2}{|c|}{ Max } & \multicolumn{2}{|c|}{ SD } \\
\hline & 1 & 2 & 1 & 2 & 1 & 2 & 1 & 2 \\
\hline Body height & 156.3 & 156.4 & 143 & 133.0 & 168 & 171.0 & 7.1 & 11.9 \\
\hline Body mass & 67.1 & 67.1 & 38.9 & 37.1 & 104.7 & 104.0 & 17.5 & 20.5 \\
\hline BMI & 27.5 & 26.9 & 15.7 & 19.1 & 47.1 & 38.6 & 7.1 & 6.0 \\
\hline$\% F A T$ & 29.2 & 30.0 & 5.8 & 9.2 & 43.9 & 50.6 & 10.0 & 12.9 \\
\hline WC & 88.0 & 86.8 & 59.0 & 65.0 & 125.0 & 118.0 & 16.9 & 17.7 \\
\hline WHR & 0.84 & 0.82 & 0.68 & 0.73 & 1.02 & 0.95 & 0.09 & 0.06 \\
\hline PWC & 97.6 & 137.6 & 26.9 & 73.0 & 215.0 & 230.0 & 40.3 & 47.0 \\
\hline $\mathrm{PWC} / \mathrm{kg}$ & 1.51 & 2.12 & 0.42 & 1.19 & 3.10 & 3.29 & 0.68 & 0.64 \\
\hline $\mathrm{VO}_{2 \max }$ & 2225.4 & 2643.5 & 1514.3 & 1984.6 & 3433.0 & 3586.0 & 417.19 & 479.7 \\
\hline $\mathrm{VO}_{2 \max } / \mathrm{kg}$ & 34.9 & 41.9 & 22.2 & 26.1 & 56.6 & 53.4 & 10.18 & 10.5 \\
\hline
\end{tabular}

Table 1. Somatic and aerobic capacity parameters of female participants

1 - age group 20-29 years; 2 - age group 30-40 years

\begin{tabular}{|c|c|c|c|c|c|c|c|c|}
\hline & \multicolumn{2}{|c|}{ Mean } & \multicolumn{2}{|c|}{ Min } & \multicolumn{2}{|c|}{ Max } & \multicolumn{2}{|c|}{ SD } \\
\hline & 1 & 2 & 1 & 2 & 1 & 2 & 1 & 2 \\
\hline Body height & 172.0 & 174.0 & 164.0 & 151.0 & 185.0 & 192.0 & 4.9 & 10.0 \\
\hline Body mass & 71.8 & 84.2 & 46.6 & 58.3 & 97.3 & 138.1 & 16.6 & 20.0 \\
\hline BMI & 24.2 & 27.4 & 17.3 & 19.1 & 32.7 & 37.9 & 5.3 & 5.4 \\
\hline$\%$ FAT & 17.7 & 22.1 & 4.7 & 6.6 & 33.3 & 41.5 & 9.3 & 8.8 \\
\hline WC & 86.7 & 95.1 & 63.0 & 72.0 & 109.0 & 126.0 & 14.5 & 15.3 \\
\hline WHR & 0.87 & 0.90 & 0.77 & 0.76 & 1.00 & 1.08 & 0.06 & 0.08 \\
\hline PWC & 168.4 & 179.4 & 77.5 & 93.8 & 355.0 & 433.0 & 70.5 & 87.0 \\
\hline PWC/kg & 2.50 & 2.14 & 0.91 & 1.23 & 6.53 & 4.90 & 1.3 & 0.0 \\
\hline $\mathrm{VO}_{2 \max }$ & 2942.5 & 3070.4 & 2030.5 & 2196.7 & 4861.0 & 5659.6 & 731.3 & 888.5 \\
\hline $\mathrm{VO}_{2 \max } / \mathrm{kg}$ & 43.5 & 37.3 & 24.0 & 24.0 & 89.5 & 67.5 & 16.6 & 10.7 \\
\hline
\end{tabular}

Table 2. Somatic and aerobic capacity parameters of male participants

1 - age group 20-29 years; 2 - age group 30-40 years 
Female participants of GR I exhibited average levels of aerobic capacity whereas GR II female subjects had high aerobic capacity. However, the difference did not reach statistical significance. Men from the same age groups exhibited average and low levels of aerobic capacity, respectively but the difference was not statistically significant either. A high percentage of study subjects had the lowest level of aerobic capacity ( $30 \%$ and $46.3 \%$ of women and men, respectively) (Fig. 1).

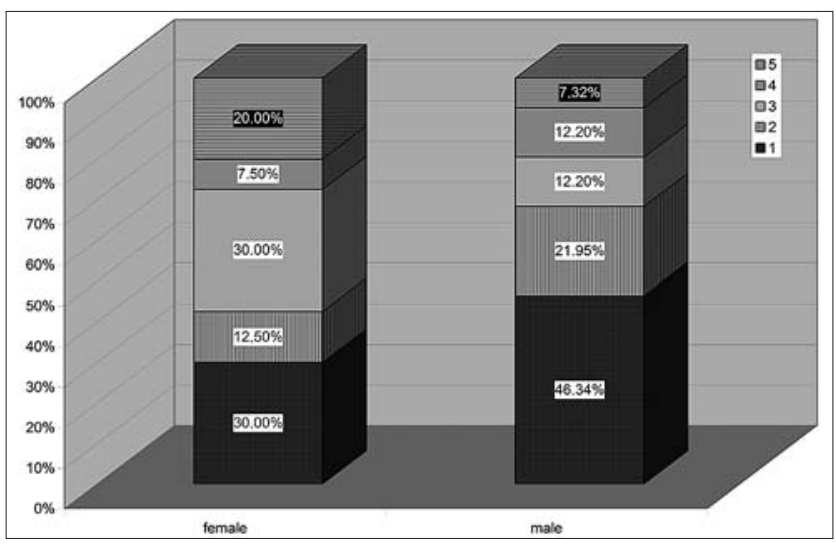

Figure 1. Aerobic capacity of male and female participants

Absolute values of $\mathrm{PWC}_{170}$ and $\mathrm{VO}_{2 \max }$ were significantly higher in men. However, relative values expressed in millilitres of oxygen per kilogram of body weight did not reveal statistically significant inter-gender differences (Fig. 2).

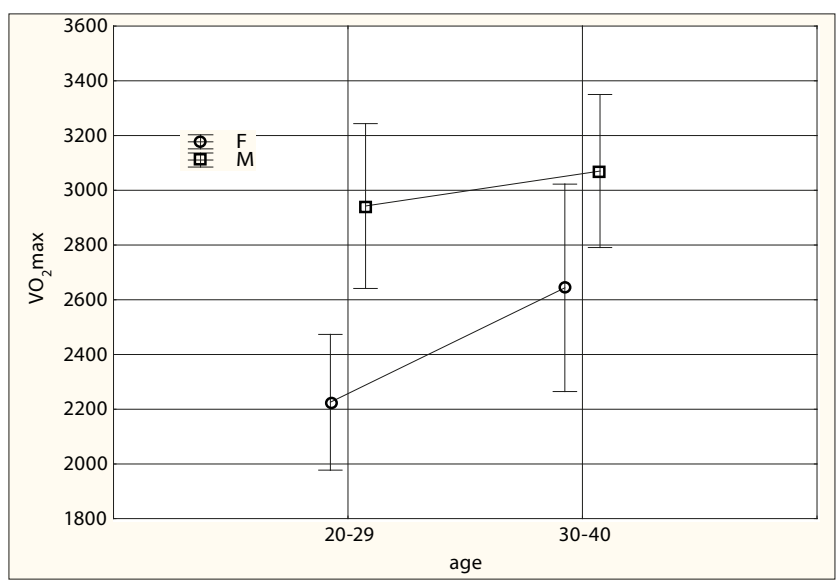

Figure 2. $\mathrm{VO}_{2 \max }$ of female and male participants

The total volume of oxygen consumption related to body weight was different for age groups. GR II women had higher $\mathrm{VO}_{2 \max / \mathrm{kg}}$ compared to GR I females whereas GR II men had lower values compared to GR I male participants. Nevertheless the differences were not statistically significant.

Aerobic capacity did not differ significantly between individuals with different degrees of intellectual disability. However, the highest percentage of participants with very low physical fitness (45\%) were among those suffering from severe intellectual disability. Study subjects with mild intellectual disability exhibited very high levels of physical fitness (23\%) (Fig.3).

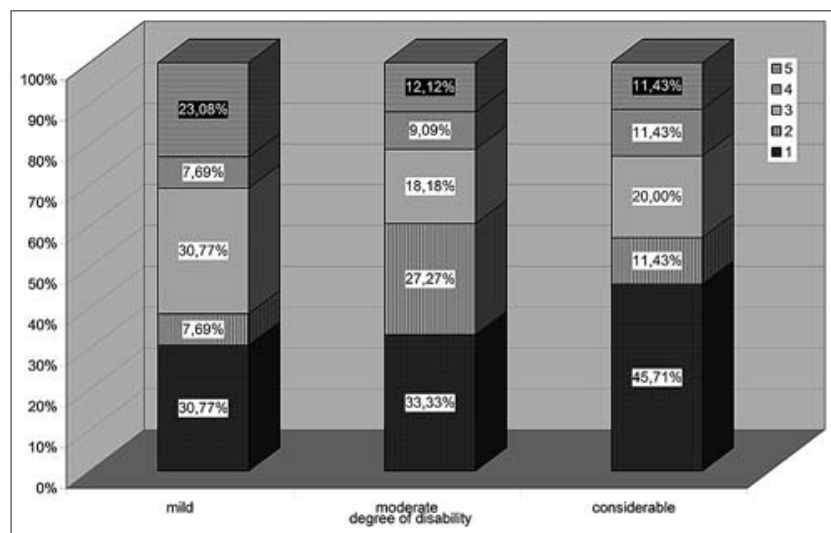

Figure 3. Aerobic capacity and the degree of intellectual disability

Physical fitness groups (levels 1 to 5) differed significantly regarding standardized somatic parameters such as body mass, waist and hips circumference, \% FAT, BMI and WHR. The highest levels of aerobic capacity were associated with the lowest standardized levels of the above mentioned parameters (Fig. 4).

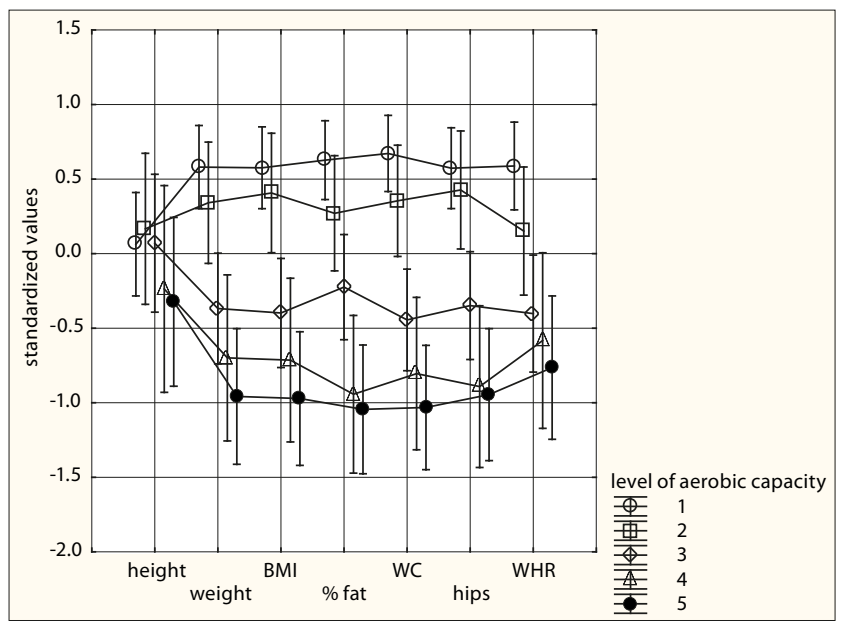

Figure 4. Somatic profile and physical fitness of study participants.

\section{DISCUSSION}

Literature review demonstrates that reports on physical fitness of the intellectually disabled are still scarce. However, available data strongly suggest lower physical fitness of the intellectually disabled compared to healthy people $[8,9,10$, 11]. Our own studies do not seem to confirm such tendency; physical fitness of our intellectually disabled participants did not differed significantly compared to that of general population. According to Astrand's classification based on the relative rate in millilitres of oxygen per kilogram of body weight, our female participants reached average or high levels of physical fitness while men's physical fitness levels were average or low. Physical fitness is determined by anthropometric parameters (body size and proportions, the percentage of muscle and fat tissue in body mass), cardiac output, arteriovenous oxygen difference, blood volume and hemoglobin concentration. All these parameters are usually better in males. Hence, men are better predisposed and have better adaptive capacity to physical effort. Maximal oxygen consumption is by $20-30 \%$ higher in men compared 
to women of the same age. The difference decreases to around $17 \%$ when expressed per kilogram body weight. In the case of lean body mass, the difference only amounts to approximately $5-11 \%$. Our results are consistent with these findings since only absolute values of $\mathrm{PWC}_{170}$ and $\mathrm{VO}_{2 \max }$ were significantly higher in men, which seems to have resulted from masculine body type. Aerobic capacity expressed per kilogram body weight did not differ between genders.

Our investigations did not reveal any relationship between the degree of intellectual disability and the level of physical fitness. Such findings are consistent with the results of [8]. The authors also found a correlation between body mass and $\mathrm{PWC}_{170}$ as well as between body mass and VO2max, which, in turn, is consistent with our results. Physical fitness parameters correlated positively with body fat percentage which is in agreement with the results of Salaun et al [11] and, at the same time, confirms the trends characteristic of the general population.

The effects of sports and recreation training on physical fitness levels are well-recognized. Klimek [12] observed that aerobic capacity of Polish cross-country skiers with mild intellectual disability did not differ from that of perfectly healthy competitors. Problems which occur in intellectually disabled skiers during competition are mainly related to the psychical sphere, eg., inability to control skiing pace, work economy and tactical strategies implementation.

The beneficial effect of physical exercise on the level of physical fitness also results from changes in body mass components, which also applies to the population of intellectually disabled individuals. Elmahgoub et al. [13] found that BMI and fat mass reduction were associated with lean body mass increase, lipid profile improvement and physical fitness enhancement in intellectually disabled adolescents who had participated in physical exercise. Our findings confirm the relationship between physical fitness and somatic parameters. High aerobic capacity was observed in those intellectually disabled individuals who had relatively high BMI but low body fat percentage. Somatic profile of individuals with high aerobic capacity is characterized by low standardized values of body mass, BMI, body fat percentage, waist and hip circumference, and WHR.

Physical exercise also helps delay the decline of the adaptive potential of the human body to exercise associated with the aging process. It has been estimated that physical fitness reaches its maximum at the age of 20-25 years, and then gradually declines by approximately $0.8-1 \%$ a year. However, regular participation of young people in physical activity not only increases its peak level but also slows down the decline to around $0.5 \%$ yearly. Intellectually disabled individuals exhibit more intensive and dynamic drop in physical fitness as confirmed by Graham et al. [14], who re-examined muscle endurance and strength, cardiovascular efficiency and body mass composition in intellectually disabled adults and the control participants 13 years after the initial study. It is believed that changes to the locomotor and cardiovascular systems of the middle-aged do not limit human ability to exercise. However, the changes accelerate in the 5th, 6th and 7 th decades of life. This might have been the reason why the age of our study subjects (20 to 40 years) did not cause significant differences between physical fitness parameters of our study subjects.

\section{CONCLUSIONS}

1. A large sample of the study population (30\% female, $46.3 \%$ male) showed very low levels of aerobic capacity.

2. Our investigations did not demonstrate a relationship between physical fitness and age or the degree of intellectual disability. Gender turned out to be a differentiating factor but only for the absolute PWC170 and VO2max.

3. The level of physical fitness was significantly related to somatic parameters including body mass, waist and hips circumference, percentage of body fat, BMI and WHR.

\section{REFERENCES}

1. Finlayson J, Cooper S, et al. Understanding predictors of low physical activity in adults with intellectual disabilities. J Appl Res Intelleck. 2009; 22 .

2. Celebańska D, Mięsok G. Wybrane elementy stylu życia osób niepełnosprawnych intelektualnie. Zeszyty Metodyczno-Naukowe AWF im. J. Kukuczki, Katowice 2011; 30 (in Polish).

3. Salaun L, Berthouze-Aranda S. Physical fitness and physical activity in adolescents with intellectual disabilities in France. Science \& Sports $2011 ; 26$ (4)

4. Salvador EP, Florindo AA, Reis R. Physical activity and its relationship with perceived environment among adults living in a region of low socioeconomic level. J Phys Act Health 2012; 11.

5. Hilgenkamp TI, Reis D, et al. Physical activity levels in older adults with intellectual disabilities are extremely low. Res Dev Disabil. 2012; 33(2).

6. McNaughton SA, Crawford D, et al. Understanding determinants of nutrition, physical activity and quality of life among older adults: the wellbeing, eating and exercise for a long life (WELL) study. Health Qual Life Outcomes. 2012; 10(1).

7. Astrand P, Rohdahl K. Textbook of Work Physiology. McGraw-Hill Book Company, New York 1977.

8. Yoshizawa S, Ishizaki T, Honda H. J Hum Ergol 1975; 4 (1).

9. Yokoyama T, Masayasu S, Fuchi T, Nakayama T, Tanaka H. Characteristics of energy metabolizm in males with mental retardation. J Nutr Sci Vitaminol. 1998; 44 (1).

10. Baynard T, Pitetti K, Guerra M, Unnithan V, Fernhall B. Age-related changes in aerobic capacity in individuals with mental retardation: a 20-yr review. Med Sci Sports Exerc. 2008; 40 (11).

11. Salaun L, Berthouze-Aranda S. Physical fitness and fitness in adolescents with intellectual disabilities. J Appl Res Intellect Disabil. 2012; 25 (3).

12. Klimek A.T, Frączek B. Physical capacity of mentally disabled crosscountry skiers and healthy effects of their sport activity. Phys Educ Sport. 2004; 48 .

13. Elmahgoub S, Lambers S, Stegen S, van Laethem C, Cambier D. The influence of combined exercise training on incides of obecity, physical fitness and lipid profile in overweight and obese adolescents with mental retardation. Eur J Pediatr. 2009; 168 (11).

14. Graham A., Reid G. Physical fitness of adults with intellectual disability: a 13-year follow-up study. Res Q Exerc Sport. 2000; 71(2). 